\title{
Source-to-target simulation of simultaneous longitudinal and transverse focusing of heavy ion beams
}

\author{
D. R. Welch, ${ }^{1}$ J. E. Coleman, ${ }^{2}$ P. A. Seidl, ${ }^{2}$ P. K. Roy, ${ }^{2}$ E. Henestroza, ${ }^{2}$ E. P. Lee, ${ }^{2}$ A. B. Sefkow, ${ }^{3}$ E. P. Gilson, ${ }^{3}$ \\ T. C. Genoni, ${ }^{1}$ and D. V. Rose ${ }^{1}$ \\ ${ }^{1}$ Voss Scientific, Albuquerque, New Mexico 87108, USA \\ ${ }^{2}$ Lawrence Berkeley National Laboratory, Berkeley, California 74720, USA \\ ${ }^{3}$ Princeton Plasma Physics Laboratory, Princeton, New Jersey 08543-0451, USA
}

(Received 8 February 2008; published 9 June 2008)

\begin{abstract}
Longitudinal bunching factors in excess of 70 of a $300-\mathrm{keV}, 27-\mathrm{mA} \mathrm{K}^{+}$ion beam have been demonstrated in the neutralized drift compression experiment [P. K. Roy et al., Phys. Rev. Lett. 95, 234801 (2005)] in rough agreement with particle-in-cell source-to-target simulations. A key aspect of these experiments is that a preformed plasma provides charge neutralization of the ion beam in the last one meter drift region where the beam perveance becomes large. The simulations utilize the measured ion source temperature, diode voltage, and induction-bunching-module voltage waveforms in order to determine the initial beam longitudinal phase space which is critical to accurate modeling of the longitudinal compression. To enable simultaneous longitudinal and transverse compression, numerical simulations were used in the design of the solenoidal focusing system that compensated for the impact of the applied velocity tilt on the transverse phase space of the beam. Complete source-to-target simulations, that include detailed modeling of the diode, magnetic transport, induction bunching module, and plasma neutralized transport, were critical to understanding the interplay between the various accelerator components in the experiment. Here, we compare simulation results with the experiment and discuss the contributions to longitudinal and transverse emittance that limit the final compression.
\end{abstract}

DOI: 10.1103/PhysRevSTAB.11.064701

PACS numbers: 29.27.-a, 52.65.Cc, 52.65.Rr

\section{INTRODUCTION}

Heavy ion fusion (HIF) and ion-driven warm dense matter (WDM) physics applications require short duration, high power ion pulses delivered to small radius targets [13]. Conventional heavy ion beam sources have been developed that provide modest current, low emittance, and long duration ion beam pulses. To significantly heat material, these pulses then require longitudinal compression and transverse focusing. Neutralized drift compression (NDC) of these intense space-charge-dominated ion beams makes use of a temporal velocity tilt and neutralizing background plasma to achieve short pulse lengths and small radial spot. In both HIF and WDM scenarios, transverse and longitudinal focusing forces are applied to the individual ion beams outside of the target chamber. To achieve the desired isochoric heating, the ion beam energy must be transported to the target and deposited in a small spot before hydrodynamic disassembly. The physics of neutralized transport, in particular, the feasibility of achieving a small transverse spot size in the presence of neutralizing plasma via neutralized ballistic transport, has been studied with simulations showing that small spots could be achieved with plasma neutralization [4-6]. The neutralized transport experiment [7-10] subsequently demonstrated that an initially unneutralized beam of several $\mathrm{cm}$ radius can be compressed transversely to $\leq 1 \mathrm{~mm}$ radius when plasma neutralization is applied in quantitative agreement with simulation $[11,12]$.
Longitudinal compression is initiated by imposing a linear head-to-tail velocity tilt to a drifting beam. In the presence of a perfectly neutralizing plasma, the compression is only limited by the accuracy of the applied velocity tilt and the ion longitudinal temperature [13]. Longitudinal compression factors of 70 have been demonstrated on the neutralized drift compression experiment (NDCX) [14,15].

Here, we make use of an integrated numerical simulation capability to model the simultaneous longitudinal and transverse compression of an ion beam. The simulations include models for ion acceleration and magnetic transport to achieve NDC and focusing of an ion beam in a dense background plasma. Previous documented simulation comparisons with data [9-12] assumed a beam temperature exiting the magnetic transport section, while no such assumption is made here except for the ion source temperature at the anode. These simulations were carried out using the LSP particle-in-cell (PIC) code [16]. The enabling algorithms include a fast implicit electromagnetic solver and energy conserving particle advance [17] that maintains the relatively low prefill plasma temperature $(\sim 3 \mathrm{eV})$ avoiding the usual problems of numerical heating on the computational grid. With these algorithms we can efficiently explore the tightly coupled physics of ion acceleration, compression, and focusing of both recent and planned experiments on the NDCX. The integrated simulations provide the opportunity to absolutely benchmark simulation against experiment and reveal important interactions between the various system components [e.g., diode, mag- 
netic transport, induction bunching module (IBM)] and the neutralizing plasma parameters on the beam focus. For example, the simulations can be used to understand the constraints on the accuracy of the applied voltage waveforms in the diode and IBM by examining relative changes in the final ion beam spot size.

This paper is organized as follows. The optimized NDCX simultaneous focus design is presented in Sec. II along with a simulation of that design. The limiting physics of longitudinal compression relating to longitudinal beam temperature is discussed in Sec. III with each limiting factor treated individually. Factors limiting the transverse focal spot size including emittance growth in magnetic transport section, aberration from the applied tilt waveform and plasma density are presented in Sec. IV. In Sec. V, we compare experimental data from the NDCX experiment with the integrated simulations. The summary and conclu-

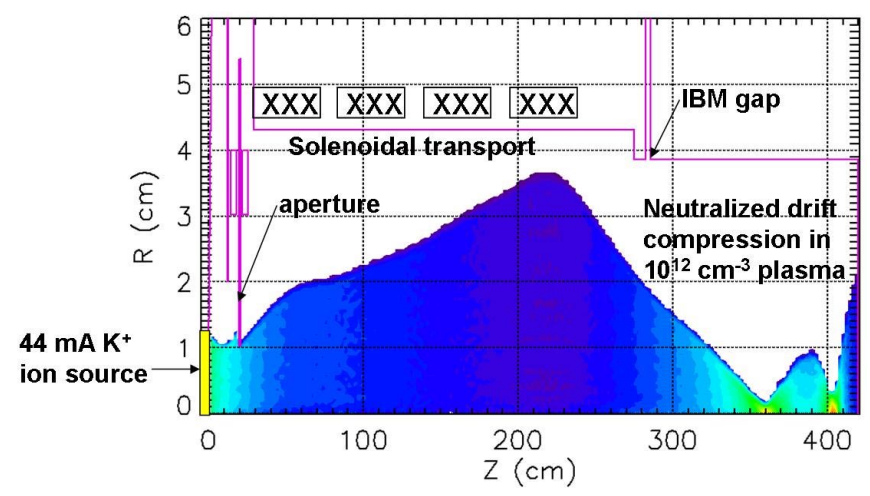

FIG. 1. (Color) The NDCX simulation geometry is shown with the beam density near the time of peak simultaneous compression. From left to right, the ion source, diode, magnetic transport, IBM gap, and plasma regions are shown. sion are found in Sec. VI. In the Appendix, a procedure for determining the optimum tilt waveform for axial compression is discussed.

\section{INTEGRATED SIMULATION OF SIMULTANEOUS FOCUS}

The NDCX experimental setup in Fig. 1 shows the position of the thermionic $0.1-\mathrm{eV} \mathrm{K}^{+}$ion source [18] at the far left $(z=0)$, diode region, solenoidal transport section, IBM gap, and finally neutralized transport and focusing $(z=415 \mathrm{~cm})$. Before analyzing the impact of these individual transport elements on simultaneous focus, we first present a $2 \mathrm{D}$ simulation using the experimentally measured diode voltage [average of 5 shots shown in Fig. 2(a)] and IBM waveform [see Fig. 3(a)]. The IBM waveform is relatively short, compressing $<200 \mathrm{~ns}$ out of the $>6 \mu$ s beam pulse. We assume a uniform $9.7 \mathrm{~mA} / \mathrm{cm}^{2}$ $\mathrm{K}^{+}$ion flux out to $1.2-\mathrm{cm}$ radius giving an initial $44 \mathrm{~mA}$ ion current in the diode. A $1-\mathrm{cm}$ radius aperture at $z=$ $20 \mathrm{~cm}$ scrapes off the beam edge leaving $27 \mathrm{~mA}$ in the apertured beam. Just upstream of the IBM gap $(z=$ $275 \mathrm{~cm}$ ), the beam has a calculated $0.01-\mathrm{eV}$ longitudinal temperature and $0.08-\pi$-mm-mrad transverse normalized emittance. The maximum fields in the four $44-\mathrm{cm}$ long, 5.8 -cm radius solenoids $(2.6,1.0,0.95,2.06 \mathrm{~T})$ establish a $2-\mathrm{cm}$ radius, -30 - $\mathrm{mrad}$ angle beam envelope at $z=$ $275 \mathrm{~cm}$ before entering the IBM gap without losing ions to the outer transport wall (assuming a Gaussian thermal beam distribution at the source). Ions lost to the wall in sufficient number would produce via secondary electron emission an undesirable electron cloud which degrades the beam quality by creating a time-dependent nonuniform partial neutralization of the beam.

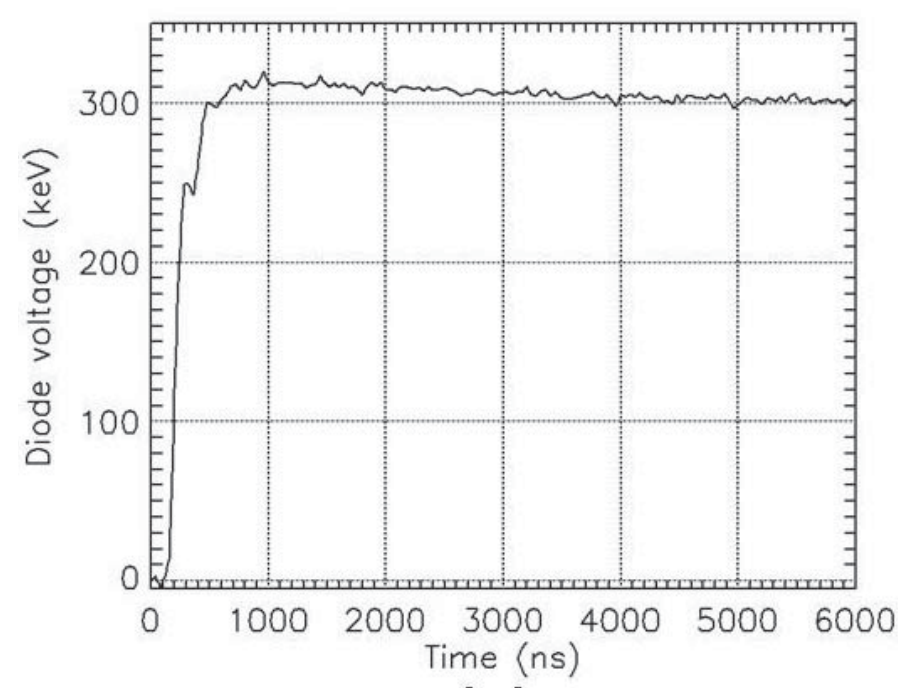

(a)

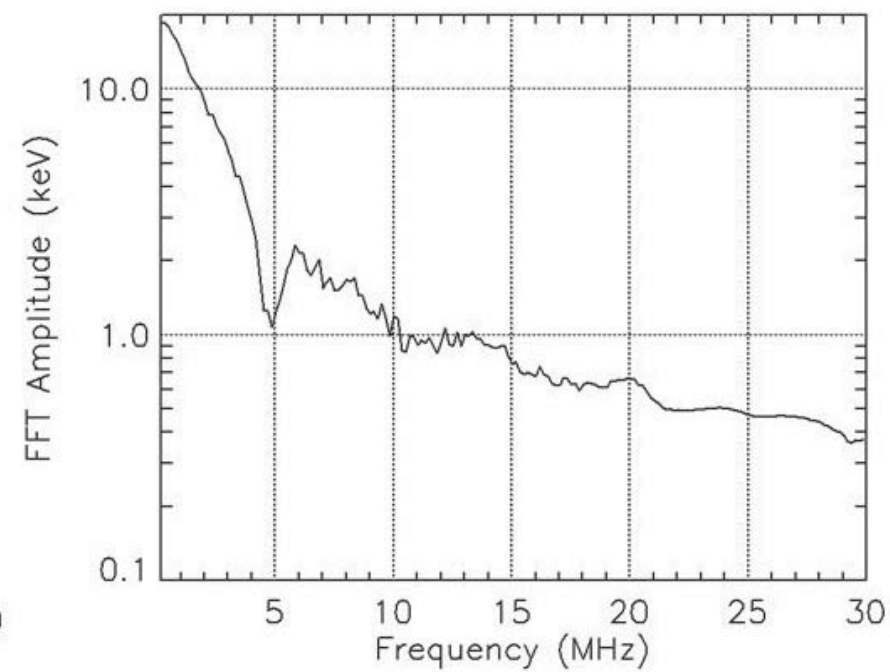

(b)

FIG. 2. A measured 5-shot average of the NDCX Marx voltage is shown in (a) with a fast Fourier transform of the waveform in (b). 


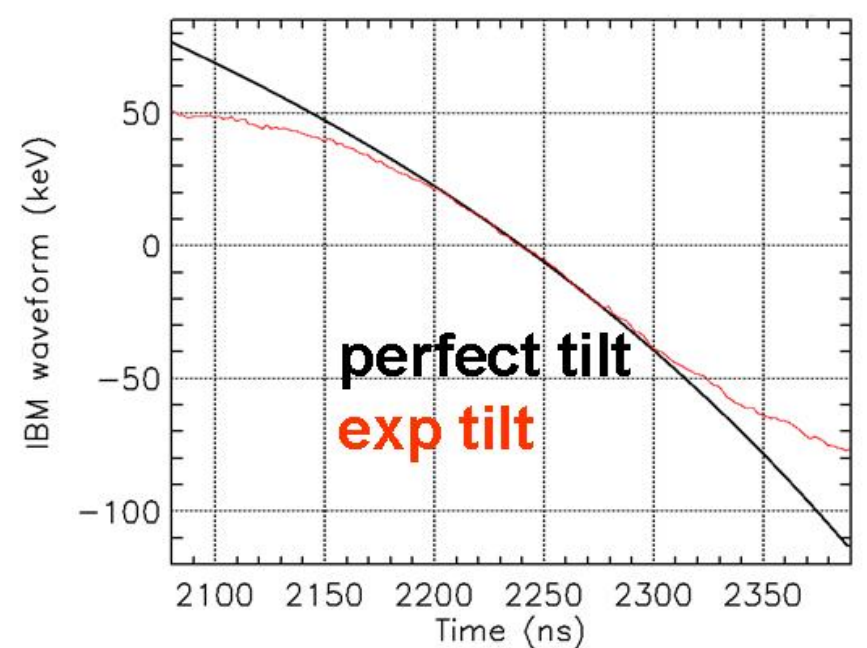

(a)

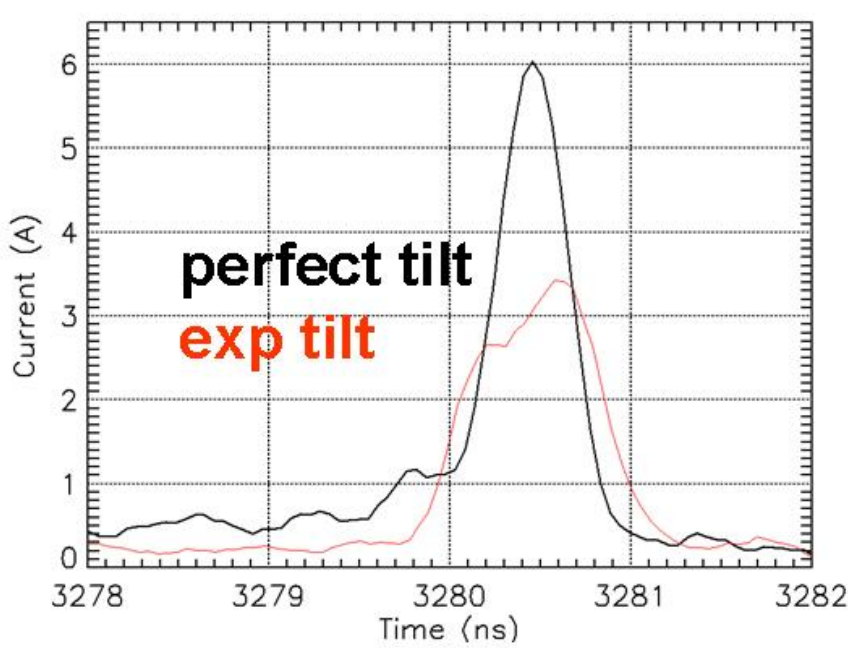

(b)

FIG. 3. (Color) In (a) the experimental and ideal IBM voltage waveform are plotted. The resulting beam currents at $z=415 \mathrm{~cm}$ are shown in (b). The transported current is $27 \mathrm{~mA}$.

The IBM waveform is chosen to optimize the head-totail energy ramp that maximizes the axial compression ratio $\mathrm{CR}=I_{\max } / I_{0}$, where $I_{\max }$ and $I_{0}$ are the maximum current at the $z=415 \mathrm{~cm}$ plane and initial beam current (alternatively, $\mathrm{CR}=t_{p 0} / t_{p \min }$ where $t_{p 0}$ and $t_{p \min }$ are the initial and minimum temporal pulse width of the compressed beam). The ideal waveform shape is discussed in Sec. III. The large incoming beam angle is chosen to counteract the defocusing produced by the IBM waveform also discussed in Sec. III and focus transversely also at the $415-\mathrm{cm}$ plane. At $z=310 \mathrm{~cm}$, the beam space charge is neutralized in a uniform $3-\mathrm{eV}, 10^{12}-\mathrm{cm}^{-3}$ density plasma. In this simulation, a reduced-description plasma model is assumed in which the plasma currents are calculated from the Ohm's Law; $\mathbf{J}=\sigma\left(\mathbf{E}+\mathbf{v}_{\mathbf{p}} \times \mathbf{B}\right)$, where $\sigma$ is the scalar conductivity and $\mathbf{v}_{\mathbf{p}}$ is the plasma electron velocity. This model provides a fast and self-consistent model of the neutralizing effect of the background plasma on the ion beam that is particularly useful in the design phase. The sensitivity of the final beam compression to the plasma density is addressed more carefully in simulations that use a fully kinetic treatment of the plasma in Sec. IV.

The beam envelope is shown in Fig. 1 after $3.2 \mu \mathrm{s}$ (80 ns before peak simultaneous focus at the $z=415 \mathrm{~cm}$ plane). At this time, the compressing beam pulse is approaching its peak density of $9 \times 10^{11} \mathrm{~cm}^{-3}$ giving a total density compression factor of 8000 relative to the beam density entering the IBM gap. The longitudinal compression peaks at $\mathrm{CR}=120$ with a transverse spot $a_{s}=1.3 \mathrm{~mm}$ [edge radius given by $\left(2\left\langle r^{2}\right\rangle\right)^{1 / 2}$, where $\left\langle r^{2}\right\rangle$ is the rms of the beam radius]. A target placed at the focus would receive peak deposition energy fluence of $0.046 \mathrm{~J} / \mathrm{cm}^{2}$. In the next two sections, we explore the physics that limits the compression in both planes.

\section{LIMITING PHYSICS OF AXIAL COMPRESSION}

We now examine the limiting physics to axial compression of an ion beam of energy $E_{i}$ given a head-to-tail velocity tilt. For a perfectly neutralized beam, the timedependent velocity function at a particular plane that produces a perfect beam longitudinal compression at a downstream distance $L$ is given by

$$
v(t)=\frac{v(0)}{1-\frac{v(0) t}{L}}
$$

where $v(0)$ is the velocity of the pulse at $t=0$. The IBM voltage waveform producing this ideal tilt for beam ion mass $m_{i}, V(t)=\frac{1}{2} m_{i} v(t)^{2}-E_{i}$, is compared with the measured IBM waveform in Fig. 3(a). In the NDCX experiment, the voltage waveform required to produce the velocity profile in Eq. (1) was approximated with an IBM consisting of 14 independently driven magnetic cores [14]. For a $300-\mathrm{keV} \mathrm{K}^{+}$beam, in the linear regime (small ratio of tilt velocity to incoming beam velocity) the required tilt voltage ramp is roughly $70 / L \mathrm{kV} / \mathrm{ns}$ or $0.7 \mathrm{kV} / \mathrm{ns}$ for a $1-\mathrm{m}$ drift. Note that the measured voltage waveform follows the ideal waveform given by Eq. (1) for $120 \mathrm{~ns}$ which corresponds to the effective $t_{p 0}$ calculated from the amount of charge longitudinally compressed in a ballistically transported beam. IBM waveforms on NDCX have produced measured $t_{p 0}$ as high as $240 \mathrm{~ns}$.

Because of the intrinsic ion source temperature and accumulated errors in the transport system, the beam ion velocities will have some characteristic thermal longitudinal velocity $\boldsymbol{v}_{\|}=\left(k T_{\|} / m_{i}\right)^{1 / 2}$ that limits the pulse length ( $2 \times$ rms width) achievable to roughly 


$$
t_{p \min }=\frac{L}{c}\left[\frac{m_{i} c^{2}}{E_{i}}\right] \sqrt{\frac{T_{\|}}{m_{i} c^{2}}}
$$

where $c$ is the speed of light. This beam frame temperature translates into the laboratory frame coordinates as energy variation $\Delta E=\left(2 E_{i} T_{\|}\right)^{1 / 2}$. Previous measurements of $t_{p \text { min }}$ have been $2-4 \mathrm{~ns}$ on NDCX with $E_{i}=300 \mathrm{keV}$ $\mathrm{K}^{+}$ions and $L=130 \mathrm{~cm}[14,15]$. This pulse width implies a total $T_{\|}=1 \mathrm{eV}$ or $\Delta E \sim 1 \mathrm{keV}$ and energy variation $\Delta E / E_{i} \sim 0.003$ for NDCX.

Several factors influence $T_{\|}$and limit $t_{p \min }$ (and CR). These include diode voltage and IBM waveform stability, instabilities such as the beam-plasma two stream and single species Harris-like mode in the magnetic transport section. We address these effects below.

\section{A. Beam longitudinal cooling in the diode}

The thermionic ion source used on NDCX has roughly $0.1-\mathrm{eV}$ temperature [18]. The calculated beam parameters just upstream of the IBM are $T_{\|} \sim 0.01 \mathrm{eV}$ with a 0.08 - $\pi$-mm-mrad normalized emittance $\left(T_{\perp} \sim 0.2 \mathrm{eV}\right)$. The very cold axial temperature is a result of the conservation of longitudinal emittance in the diode (with applied voltage $V$ ) that reduces the beam temperature from that of the anode source $T_{s}$ in the $1 \mathrm{D}$ limit as described by $k T_{\|}=$ $\left(k T_{s}\right)^{2} /(2 \mathrm{eV})$ [19]. Ideally, this cooling would reduce the NDCX beam to $1.7 \times 10^{-8} \mathrm{eV}$. Such a cold beam, given a perfect tilt and focusing angle, would yield a 0.1-ps pulse. The integrated 2D simulation calculates $T_{\|} \sim 0.01 \mathrm{eV}$ or $\Delta E=100 \mathrm{eV}$ at the entrance to the IBM gap that would translate into $t_{p \min }=0.3 \mathrm{~ns}$ with the ideal IBM waveform. In these simulations, the time step $\Delta t$ is already much smaller than necessary to resolve the beam-plasma frequency $\omega_{p b}=\left(4 \pi n_{b} \mathrm{e}^{2} / m_{i}\right)^{1 / 2}$, where $n_{b}$ is the beam density (typical numbers are $\Delta t=0.025 \mathrm{~ns}$ and $1 / \omega_{p b}=$ $450 \mathrm{~ns}$ ). Because of the discrete sampling of the electric field as the ions traverse the diode gap, the calculated $T_{\|}$is limited by the ratio of $\Delta t$ to the ion acceleration time across the gap $t_{a}$ with $\Delta E / E_{i} \sim 1 / 2 \Delta t / t_{a}$. In these simulations, $\frac{1}{2} \Delta t / t_{a}=1.5 \times 10^{-4}$ which is of the same order of the calculated $\Delta E / E_{i}$. Reducing $\Delta t$ by half decreased $T_{\|}$by $40 \%$ just downstream of the diode $(z=28 \mathrm{~cm})$. Just upstream of the IBM gap $(z=275 \mathrm{~cm})$, however, $T_{\|}$had increased to the $0.01 \mathrm{eV}$ value calculated in the larger $\Delta t$ simulation. This smaller time step yielded (within 10\%) the same CR even when using the ideal IBM waveform in both simulations.

\section{B. Beam space-charge depression}

A physical limitation on $\Delta E$ results from the beam space-charge depression in a long drift tube of radius $R_{w}$,

$$
\varphi_{\mathrm{sc}}(r)=\left[1-\left(\frac{r}{a}\right)^{2}+2 \log \frac{R_{w}}{a}\right] \frac{\nu}{\beta} m_{e} c^{2},
$$

where $a$ is the beam radius, $m_{e}$ is the electron mass, $\nu$ is the Budker number (beam current normalized by $17 \mathrm{kA}$ ), and $\beta$ is beam velocity normalized by the speed of light $c$. Integrating over the beam envelope assuming a uniform transverse charge distribution, the energy spread about the mean space-charge depression is

$$
\Delta E_{\mathrm{sc}}=12^{-1 / 2} \frac{\nu}{\beta} m_{e} c^{2} .
$$

Ion transverse thermal motion or phase mixing of ion orbits while $a$ is changing can increase $\Delta E$ to some fraction of $\Delta E_{\mathrm{sc}}(58 \mathrm{eV}$ for the $27-\mathrm{mA}$ beam). As the ions reach the plasma which is roughly at the potential of the drift tube, they gain back the $\varphi_{\text {sc }}$ plus whatever variation gained due to phase mixing. In the vacuum transport distance between the IBM gap and the plasma, the effective $T_{\|}=$ $\left(\Delta E_{\mathrm{sc}}\right)^{2} /\left(2 E_{i}\right)$ contains the full spread in $\Delta E_{\mathrm{sc}}$ and will have an effect on $t_{p \text { min }}$ even in the absence of phase mixing. For $27-\mathrm{mA}$ current, $T_{\|}=0.006 \mathrm{eV}$, roughly the minimum value calculated in the integrated simulation and roughly the minimum beam longitudinal temperature we can expect. Correspondingly, $t_{p \min }=0.22 \mathrm{~ns}$ is the minimum pulse width with a perfect IBM waveform $(\mathrm{CR} \approx 500)$.

\section{Velocity spread due to focusing angle}

After passing through the IBM gap, the beam is left by design with the proper focusing angle to coincide with the longitudinal focus that scales with radius $r$. The resulting angular spread (or equivalently path length spread) adds to the effective $T_{\|}$of the beam. This additional spread is easily calculated assuming a small focusing angle $\theta$ at $r=a$ giving $T_{\| \text {foc }}=\left(m_{i} c^{2} \beta^{2} \theta^{4}\right) / 24$. For a characteristic 15 -mrad angle corresponding to $L=130 \mathrm{~cm}$ and $a=$ $2 \mathrm{~cm}, T_{\| \text {foc }}=0.0006 \mathrm{eV}$ which is negligible. If, for example, $\theta$ were to double, however, $T_{\| \text {foc }}$ would increase by over an order of magnitude.

\section{Voltage oscillations in diode}

As discussed earlier, the effective energy variation of the beam just downstream of the IBM must be of order $0.3 \%$ to produce the measured 2-4 ns compressed pulses. The measured diode voltage produced by the Marx has variations of order $1 \%$ as shown in a 5-shot average in Fig. 2. An ion bunch accelerated in the diode voltage with fluctuation periods $t_{f}$ such that $t_{a}<t_{f}<t_{p 0}$ will experience the most damage to the beam longitudinal emittance and limit longitudinal compression. Very high frequency $\left(\nu \gg 1 / t_{f}\right)$ fluctuations average out during acceleration and very low frequency $\left(\nu \ll 1 / t_{f}\right)$ fluctuations add only a small velocity tilt to the beam. If significant, this tilt can affect the plane of longitudinal focus but not the peak compression. For the $300-\mathrm{keV} \mathrm{K}^{+}$ions, both beam time scales are $100-$ $200 \mathrm{~ns}$ or $5-10 \mathrm{MHz}$ frequency which means only a very 
TABLE I. Comparison of CR with various voltage fluctuations.

\begin{tabular}{ccccc}
\hline \hline $\begin{array}{c}\text { Frequency } 1 / t_{f} \\
(\mathrm{MHz})\end{array}$ & $\begin{array}{c}\text { Amplitude } \\
\%\end{array}$ & $\begin{array}{c}\text { Focal } \\
\text { plane }(\mathrm{cm})\end{array}$ & $\begin{array}{c}\text { CR at } \\
z=412 \mathrm{~cm}\end{array}$ & $\begin{array}{c}\text { Peak } \\
\mathrm{CR}\end{array}$ \\
\hline $\mathrm{NA}$ & 0 & 412 & 120 & 120 \\
3.3 & 1 & 407 & 70 & 123 \\
5.0 & 1 & 410 & 97 & 114 \\
10 & 1 & 412 & 120 & 120 \\
\hline \hline
\end{tabular}

limited frequency band can contribute to the relevant longitudinal emittance of the bunch. Integrated simulations with applied diode voltage $V(t)=300[1+$ $\left.A \sin \left(2 \pi t / t_{f}\right)\right] \mathrm{kV}$ were performed with results shown in Table I. A $1 \%$ voltage variation at any frequency is clearly not sufficient to grossly affect peak CR. As expected, only the $5 \mathrm{MHz}$ variation reduced compression and only by $5 \%$. The 3.3-MHz frequency actually increased CR slightly by fortuitously improving the velocity tilt and reducing the axial focal length by $5 \mathrm{~cm}$. The relative shift in the plane of maximum longitudinal compression from that of the nominal compression plane with flat voltage $(z=412 \mathrm{~cm})$ is seen in Table I. In the NDCX experiments, these shifts can lead to a spread in $\mathrm{CR}$ with the $3.3 \mathrm{MHz}$ simulation yielding $\mathrm{CR}=70$ at $z=412 \mathrm{~cm}$. For target heating experiments, the shift can add some uncertainty to the energy deposition time although the energy deposition over that time is not affected. As the IBM waveform pulse length is increased in an effort to compress a longer beam pulse, the dangerous band of frequencies will increase and voltage fluctuations will become more important.

\section{E. Beam plasma two stream}

The electrostatic beam-plasma electron two stream or Buneman instability results in longitudinal emittance $\epsilon_{\|}$ (or $T_{\|}$where $\epsilon_{\|} \sim T_{\|}^{1 / 2}$ ) growth that could limit compression. The instability is driven by the relative velocity of the beam ions and the neutralizing background plasma electrons. The asymptotic small-signal gain factor for the instability as a function of propagation distance $z$ in a plasma with electron plasma frequency $\omega_{p}$ and time into the pulse $\tau$ obtained by Briggs [20] is given by

$$
G(z, \tau)=\frac{3 \sqrt{3}}{4}\left(\frac{\omega_{p b} z}{v_{z}}\right)^{2 / 3}\left(\omega_{p} \tau\right)^{1 / 3}
$$

for beam velocity $v_{z}$. The linear growth rate has been analyzed in 1D spherical geometry for monoenergetic "focusing" beams [21]. Recent work has shown that the instability typically saturates with negligible heating to a transverse focusing beam [22]. Furthermore, theory and simulations have shown that the velocity tilt of the compressing beam also reduces the growth rate [23,24]. Unfortunately, the spatial resolution required to adequately
TABLE II. Change in longitudinal emittance with initial beam and plasma temperatures.

\begin{tabular}{ccc}
\hline \hline$T_{\|}(0)(\mathrm{eV})$ & $T_{p}(0)(\mathrm{eV})$ & $\% \varepsilon_{\|}$increase at $r=10 \mathrm{~cm}$ \\
\hline $5 \times 10^{-4}$ & 0.01 & 120 \\
0.02 & 0.01 & 78 \\
0.02 & 1 & 36 \\
0.02 & 3 & 25 \\
\hline \hline
\end{tabular}

model this instability is $\Delta x<\frac{v_{z}}{\omega_{p}}$ or roughly $0.01 \mathrm{~cm}$. This cell size is prohibitively small for a multidimensional simulation over $1 \mathrm{~m}$ in length. A series of highly spatially and temporally resolved 1D spherical LSP simulations, in which a $\mathrm{K}^{+}$ion beam is injected into a uniform plasma of temperature $T_{p}$ and number density $n_{p}$, are summarized in Table II. The beam is injected towards the axis at $r=$ $100 \mathrm{~cm}$ and diagnosed at $r=10 \mathrm{~cm}$.

Given a very cold beam longitudinal temperature of $4 \times$ $10^{-4} \mathrm{eV}$ and cold Al plasma $\left(T_{p}=0.01 \mathrm{eV}, n_{p}=\right.$ $3 \times 10^{10} \mathrm{~cm}^{-3}$ ) for an axially and transversely compressing beam with NDCX parameters (1-m focal length, 200ns ideally compressing beam pulse), the two-stream instability growth in the simulations results in modest longitudinal emittance growth. Here the longitudinal emittance increases by only a factor of 2.2. This weak growth is consistent with Eq. (5) which predicts a maximum linear growth of only 24 for these conditions. For an initially warmer beam with $T_{\|}=0.02 \mathrm{eV}$, the emittance grew $78 \%$. A simulation with the more realistic $3-\mathrm{eV}$ initial plasma temperature and $T_{\|}=0.02 \mathrm{eV}$ resulted in an increase in the beam emittance of $25 \%$. This increase in $\epsilon_{\|}$ will have an impact on compression in the NDCX experiment possibly reducing CR up to $25 \%$.

\section{F. Beam temperature anisotropy instability}

In a recent series of papers, Startsev, Davidson, and Qin [25-31] have analyzed and simulated the stability properties of intense non-neutral charged particle beams with large temperature anisotropy. They show that an electrostatic Harris-like instability [32] may develop in such beams and is a potential source of deterioration of beam quality (growth of $T_{\|}$and longitudinal beam emittance). For the 0.044-A, 300-keV K ${ }^{+}$beam of NDCX [9], we assume an average solenoidal focusing field of 1.6 T. We further assume a uniform density profile with $2-\mathrm{cm}$ edge radius. These parameters imply a highly space-charge dominated beam with normalized beam intensity parameter $s_{b}=\omega_{p b} / \omega_{f b}$ slightly less than unity [where $\omega_{f b}=$ $e B /\left(2 m_{i} c\right)$ is the transverse frequency associated with the applied focusing field $B$ in the smooth focusing approximation]. The normalized tune depression $v / v_{o}=$ $\left(1-s_{b}\right)^{1 / 2} \leq 0.1$ [see Eqs. (11)-(20) of Ref. [25] for a detailed discussion of these parameters]. For an initially 
cold beam $\left(T_{\|} \ll T_{\perp}\right)$, we expect a peak growth rate for the Harris-like mode of $\omega_{i} \leq 0.2 \omega_{f b}$ (see Figs. 2 and 7 of Ref. [27] and Fig. 1 of Ref. [28]). For this case, $\omega_{f b} \sim$ $1.97 \times 10^{6} \mathrm{~s}^{-1}$. For a 3-m drift length, $T_{\text {drift }} \sim 2.5 \mu \mathrm{s}$ which gives a maximum exponential growth factor $\omega_{i} T_{\text {drift }}<1$. Because theory predicts a dipole or $m=1$ mode to be the most virulent, we compared growth of $T_{\|}$in 2D and 3D LSP integrated simulations. Consistent with theory which predicts little growth for any mode, the 3D simulations, which used 8 azimuthal grid points enabling resolution of modes $m \leq 4$, result in no additional growth in $T_{\|}$than the 2D simulations. We therefore expect no significant growth of longitudinal beam emittance for the NDCX beam due to the Harris-like mode. We should also note that the above authors observed a threshold longitudinal temperature for initiation of the Harris-like instability of $T_{\|} / T_{\perp} \sim 0.06$ (for beams with $s_{b}$ near 1.0). (See Fig. 10 of Ref. [27] and Fig. 4 of Ref. [28].) Saturation values of $T_{\|}$ consistent with these thresholds were also observed (Fig. 5 of Ref. [30]) so that for arbitrarily long transport lengths we can expect the growth of $T_{\|}$to be bounded by $0.06 T_{\perp}$ in these cases. In our integrated NDCX simulations, we typically calculate $T_{\|}=0.05 T_{\perp}$ which is just within the unstable range. So even given sufficient drift time for the instability to develop, we still expect little growth.

\section{G. Deviation in tilt waveform from ideal}

The IBM voltage waveform, constructed for the NDCX experiment to approximate the ideal waveform derived from Eq. (1), is shown in Fig. 3(a). The experimental voltage is a very good approximation to the ideal for roughly $120 \mathrm{~ns}$ (between $t=2190$ and $2310 \mathrm{~ns}$ ). Beyond that, the experimental voltage (with roughly $3 \mathrm{kV}$ uncertainty) deviates strongly from the ideal. The longitudinal compressions in the integrated simulations using both the ideal (for $300 \mathrm{~ns}$ ) and experimental waveforms are shown in Fig. 3(b). The compression increases $84 \%$ to $\mathrm{CR}=220$ from that using the experimental waveform. This increase for the ideal waveform is consistent with the effectively longer IBM pulse width (or $t_{p 0}$ ) and more gathered beam charge.

As discussed in the Appendix, there are two sources of error that result in reduced compression or modification of $L$. First, the 25-ns transit time across the gap causes the ions to sample a range of tilt voltages. Second, between the tilt gap and the neutralizing plasma, the beam space charge becomes axially nonuniform due to the applied tilt resulting in a net electrostatic force on the beam. The ideal voltage is not perfect and does not produce the maximum possible compression from Eq. (2) $(\mathrm{CR}=500)$ without further small voltage corrections of order $3 \mathrm{kV}$. As discussed in the Appendix, these two effects can be compensated through modification of the tilt waveform and do not necessarily lead to increased beam effective temperature.

\section{FACTORS LIMITING TRANSVERSE FOCUS}

Compression or focusing of the NDCX beam in the transverse plane is complicated by the longitudinal physics. The key complications involve transport through the IBM gap which imparts a time-dependent transverse momentum to the beam (as in an Einzel lens) and the increased beam density particularly in the plasma region due to the axial compression. These effects are now addressed.

\section{A. Aberrations from IBM module}

The gap across which the IBM voltage waveform is applied acts as a simple bipotential lens, in which the radial component of the electric field is antisymmetric about the center of the gap. In the paraxial approximation, the radial electric field $E_{r}$ is also proportional to ion radius $r$. The beam envelope receives a net positive radial impulse on the upstream side of the gap [assuming a positive voltage difference $V(t)$ across the gap] and net negative radial impulse on the downstream side of the gap. For a constant voltage, the slight change in the radius of particles as they traverse the gap results in incomplete cancellation of the upstream and downstream radial forces and leads to a small net focusing effect. For NDCX, however, the ion beam is relatively slow and the gap voltage can change significantly during the 25-ns ion transit time through the lens. So the net radial forces upstream and downstream of the center of the gap do not cancel since $E_{r}$ is proportional to the time-dependent voltage. With a simple thin-lens model in which the radial forces are modeled by delta function impulses separated by the width of the gap, the timedependent change in radial momentum of a nonrelativistic ion going through the gap can be approximated by the formula [33]

$$
\begin{aligned}
\Delta v_{r} & \approx-\frac{e r}{2 m_{i} v_{z} d}\left[\Delta V\left(t_{0}\right)-\Delta V\left(t_{0}-d / v_{z}\right)\right] \\
& \approx-\frac{e r}{2 m_{i} v_{z}^{2}} \frac{d V}{d t}
\end{aligned}
$$

where $v_{z}$ is the axial velocity of the ion upstream of the gap. The change in the axial velocity and radius of the ions is neglected. So a large and negative $d V / d t$, which is required for longitudinal focusing, also has a net transverse defocusing effect. Because the ideal tilt voltage ramp is not constant, the compressing ion bunch is given an effective increase in emittance. The magnitude of this effect in the NDCX experiment can now be estimated assuming a 2-cm $285-\mathrm{keV}$ beam and the previously calculated IBM voltage ramps as $10 \mathrm{mrad}$ for the $1-\mathrm{m}$ drift length and $5 \mathrm{mrad}$ for the 2-m drift. Of course, the axial velocity is altered by the gap as well. The change in axial velocity is given by

$$
\frac{\Delta v_{z}(t)}{v_{z}}=1-\sqrt{1-\frac{2 e V(t)}{m_{i} v_{z}^{2}}},
$$

due to a change in IBM voltage $V(t)$. This axial velocity 
spread of about $\pm 10 \%$ for a $285-\mathrm{keV}$ beam from the IBM voltage waveform gives an additional $2 \mathrm{mrad}$ spread for typical parameters ( -30 -mrad incoming angle). The total effect on the beam envelope is given by

$$
a^{\prime}=\frac{v_{r 0}+\frac{a \Delta v_{r}(t)}{r}}{v_{z 0}+\Delta v_{z}(t)}
$$

where $a$ and $a^{\prime}$ are the edge radius and angle at the gap.

Ideally, a larger radius beam leads to a smaller spot $a_{s}=$ $\varepsilon_{n} L / a_{0} \gamma \beta$ for a given beam transverse emittance $\varepsilon_{n}$ and focal length $L$. The gap aberration, however, is worse for a larger radius beam. Three idealized simulations with uniform beam profiles of $1.5,2$, and $3 \mathrm{~cm}$ radius, $\varepsilon_{n}=$ $0.08 \pi$-mm-mrad and $L=130 \mathrm{~cm}$ show that the larger radius beam gives a smaller spot despite the aberration. The experimental IBM waveform was used and beam was injected at $z=275 \mathrm{~cm}$, just upstream of the IBM gap. All calculations were optimized for simultaneous focus at $z=$ $415 \mathrm{~cm}$ by adjusting the incoming angle $a^{\prime}$ at injection. The simulations with $a(275 \mathrm{~cm})=1.5,2$, and $3 \mathrm{~cm}$ had identical longitudinal compression but with 0.007, 0.019, and $0.027 \mathrm{~J} / \mathrm{cm}^{2}$ energy deposition, respectively. The ratio of the energy deposition of tilted beam to that of an untilted beam decreases with incoming radius, however, indicating that the gap aberration is becoming increasingly important. If the time-dependent aberration could be corrected, a fourfold higher deposition could be achieved.

\section{B. Beam misalignments entering IBM gap}

Misalignments of the ion source and solenoid axis can lead to offset in the beam centroid or a dipole beam motion which are magnified in the IBM gap. The impact of such an offset or motion on the beam transverse focus is briefly assessed in 3D simulations. Here, beam particles are captured at the $z=275 \mathrm{~cm}$ plane from the 2D integrated simulation as discussed in Sec. II, but they are injected in the 3D cylindrical simulation which begins at this same plane after being given a rigid offset in the $x=0$ plane of 2 and $5 \mathrm{~mm}$. The 3D simulations (which again use 8 grid positions in $\theta$ ) allow for modest offset from axis of the beam. Because we are interested only in the beam interaction in the IBM gap, we can use the reduced-description plasma model discussed in Sec. II for computational speed. The changing gap field acts to magnify any incoming beam offset. Once again for the NDCX configuration and IBM waveform, the longitudinal compression was $\mathrm{CR}=120$, however the offsets at the simultaneous focus plane are roughly doubled as seen in Fig. 4. By itself, this offset could have serious consequences for target heating if it is not systematically repeatable and the targets are small. In addition to the beam focus being driven further off axis, there is a pronounced sweep or elongation in the target plane that reduces the spot size and energy deposition from the $2 \mathrm{D}$ ideal result of $0.046 \mathrm{~J} / \mathrm{cm}^{2}$ to $0.0295 \mathrm{~J} / \mathrm{cm}^{2}$ for the 2-mm offset and $0.019 \mathrm{~J} / \mathrm{cm}^{2}$ for the 5 -mm offset. From these calculations, keeping beam offsets at the IBM gap to $<1 \mathrm{~mm}$ should lead to $<15 \%$ degradation from the $2 \mathrm{D}$ simulation result.

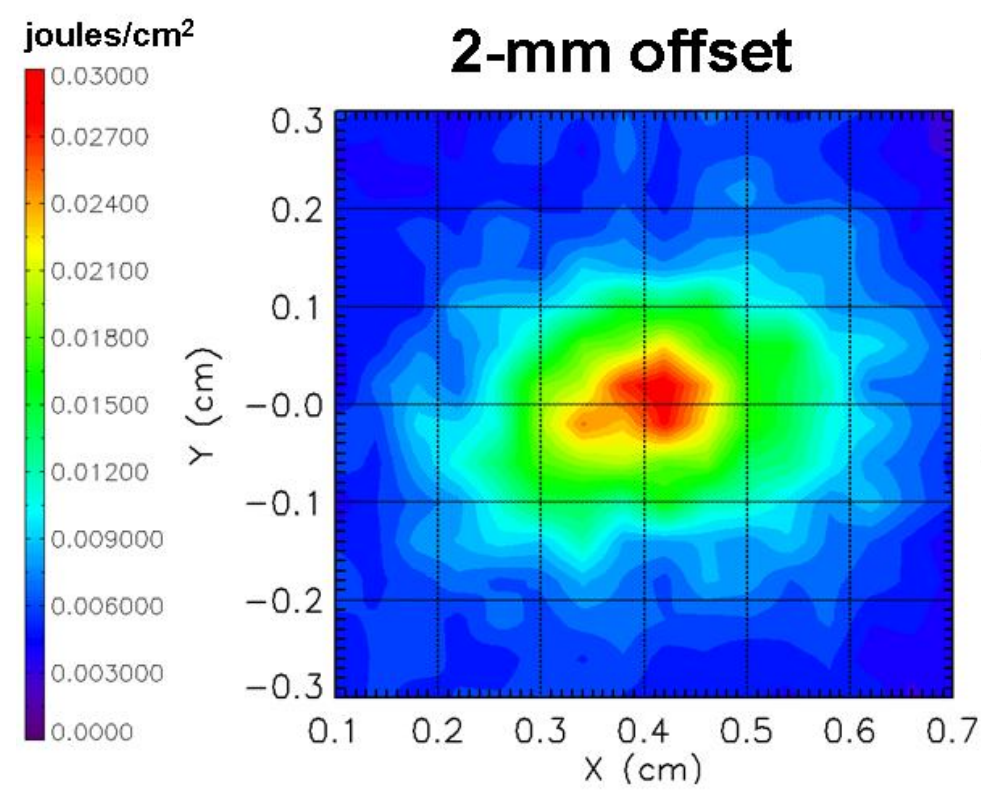

(a)

\section{5-mm offset}

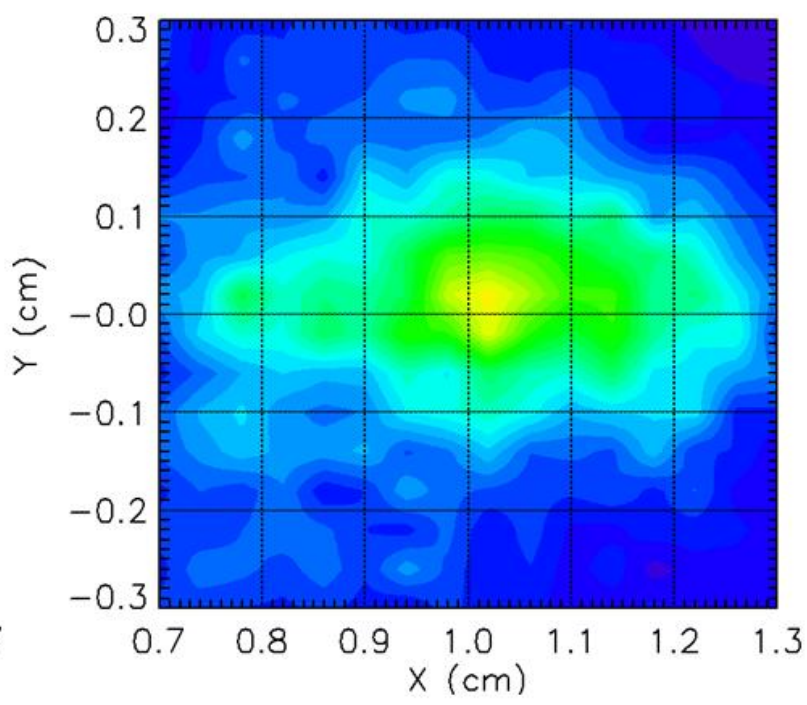

(b)

FIG. 4. (Color) The time integrated energy deposition at the target plane from the compressed beam pulse is plotted for beam offsets of (a) $2 \mathrm{~mm}$ and (b) $5 \mathrm{~mm}$. 


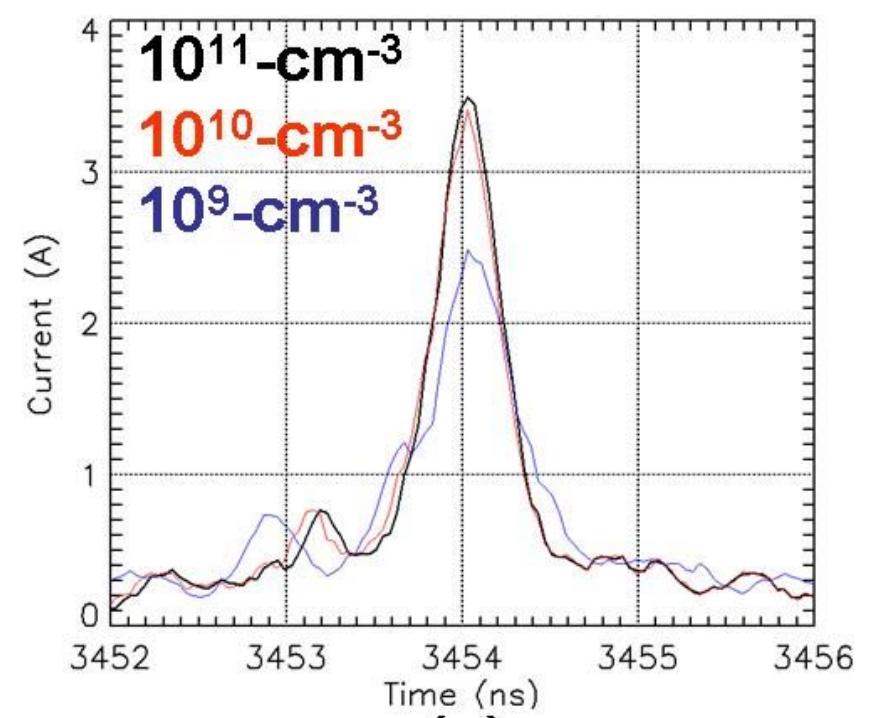

(a)

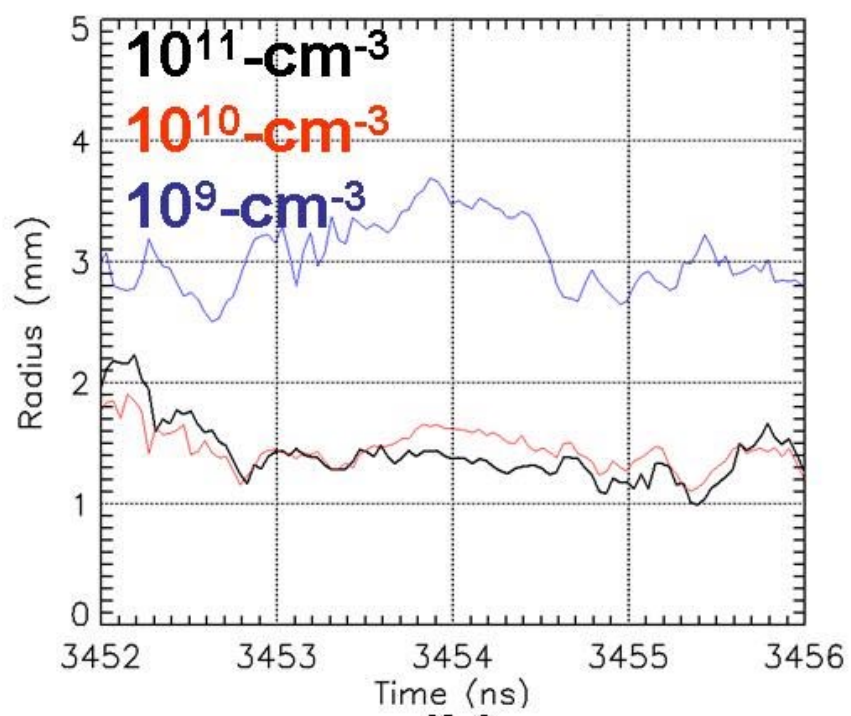

(b)

FIG. 5. (Color) In a series of kinetic plasma simulations, the (a) compressed beam current and (b) edge radius at simultaneous compression are shown for the three plasma densities as a function of time.

\section{Sensitivity of focus to plasma density}

For a purely transversely focusing beam, ion beam space charge can effectively be neutralized ( $>99 \%$ ) in the presence of a high density plasma $n_{p} \gg n_{b}$ [34]. As the beam density approaches that of the plasma, the plasma electrons will heat significantly which increases the plasma Debye length exposing a greater fraction of the beam space charge. The reduced plasma model used in Sec. II neglects electron inertia and assumes a constant density of $10^{12}-\mathrm{cm}^{-3}$. In this section, we utilize a more realistic kinetic electron-ion PIC model for the plasma and assess the impact of the plasma density ranging from $10^{9}-10^{11} \mathrm{~cm}^{-3}$ (roughly the range seen in experiments $[14,15])$ on the beam focal spot. As above, we reinject beam particles at the $z=275 \mathrm{~cm}$ plane from the integrated simulation. The beam current and edge radius at peak compression are shown in Fig. 5 for the three simulations. The results are encouraging with $10^{11}-\mathrm{cm}^{-3}$ density yielding the same compression as the simple plasma model (3.3 A and $1.3 \mathrm{~mm}$ radius). The $10^{10}-\mathrm{cm}^{-3}$ density simulation shows a slight degradation in maximum current, but the minimum radius increased to $1.7 \mathrm{~mm}$. In this simula-

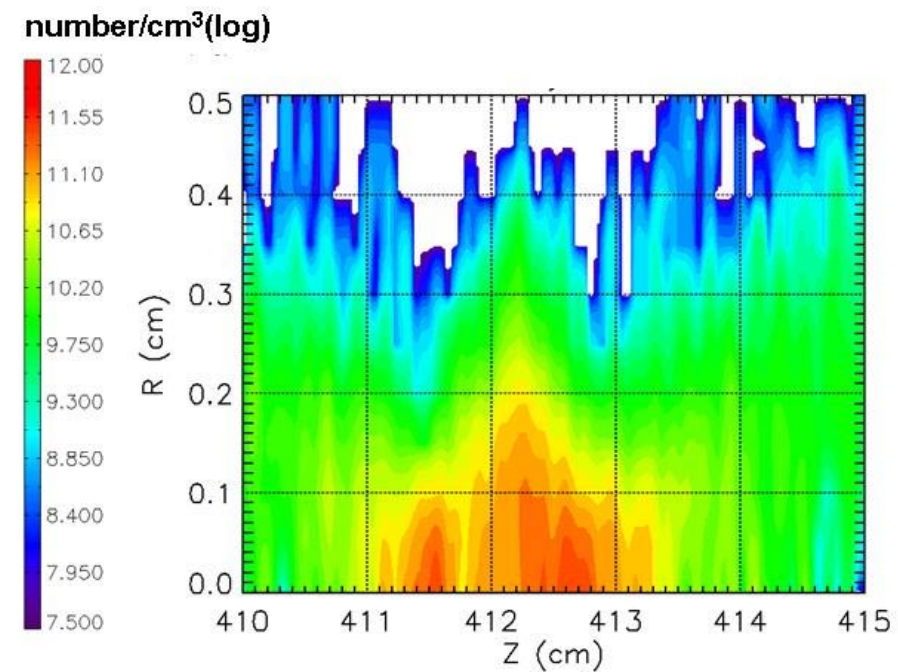

(a)

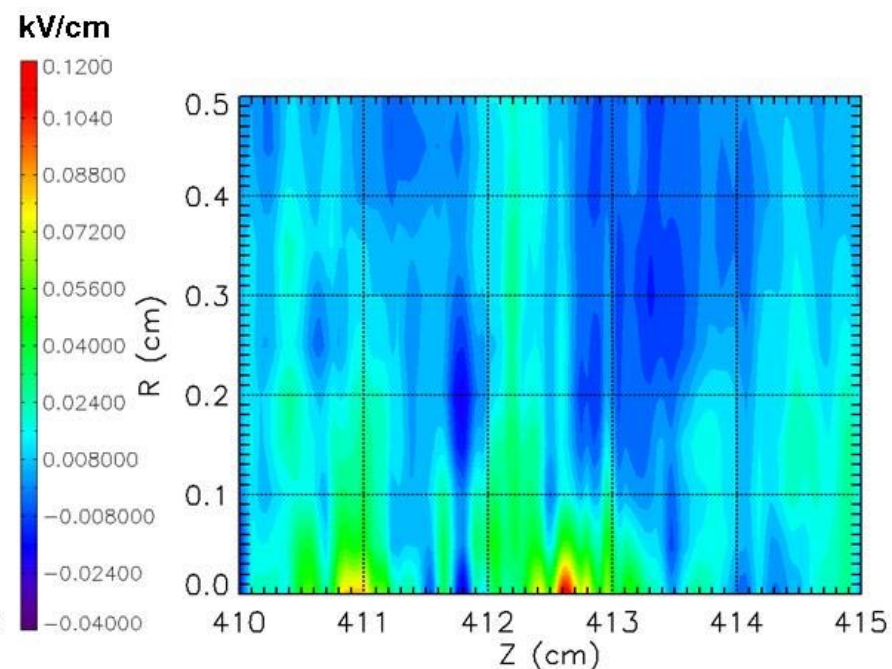

(b)

FIG. 6. (Color) The (a) beam density and (b) residual electrostatic potential are plotted just before peak compression (1900 ns) in the $10^{10}-\mathrm{cm}^{-3}$ density simulation. 
tion, the maximum beam density at focus exceeded the plasma density by nearly a factor of 80 with residual electrostatic potentials within the beam $<80 \mathrm{eV}$ as shown in Fig. 6. Note the axial potential oscillations, within the compressed beam, have roughly 2 -mm wavelength suggestive of plasma wakefields $\left(\lambda_{w f}=2 \pi v / \omega_{p}\right.$, where $v$ is the beam velocity and $\omega_{p}$ is the electron plasma frequency). These oscillations, while temporally well resolved, are spatially under resolved at the higher plasma densities with the $0.5-\mathrm{mm}$ simulation cell size. This may result in an underestimation of the coupling of the oscillations to the ion beam emittance growth. Finally, the $10^{9}-\mathrm{cm}^{-3}$ density simulation gave a $33 \%$ smaller current and a $3.5-\mathrm{mm}$ spot. Because of the strong longitudinal compression and rapidly changing beam density, adequate beam neutralization requires significantly smaller plasma density than beam density, at least for the shallow 7-mrad focusing angle. The $10^{11}-\mathrm{cm}^{-3}$ plasma density simulation calculates a 20 $\mathrm{eV}$ residual potential within the beam as it focuses giving 99.6\% charge neutralization. The beam space charge would have to be completely unneutralized for $2 \mathrm{~cm}$, given the peak 0.08 beam dimensionless perveance and $1.3-\mathrm{mm}$ radius, to strongly affect the compression ratio $\mathrm{CR}$. The maximum energy deposition at the focal plane for the $10^{9}$, $10^{10}$, and $10^{11}-\mathrm{cm}^{-3}$ plasma densities was $0.022,0.031$, and $0.034 \mathrm{~J} / \mathrm{cm}^{2}$, respectively.

\section{COMPARISON WITH NDCX MEASUREMENTS}

We now compare the simulated simultaneous compression with that measured in the NDCX experiment. In the experiment detailed in Ref. [35], the solenoids in the magnetic transport section have peak on-axis fields of $2.6,1.4,0.6$, and $2.32 \mathrm{~T}$, slightly different from the tune used in the previous section giving a smaller radial excursion in the magnetic transport section to avoid beam ion impact on the wall and resulting electron cloud effects. The plasma neutralization began $28 \mathrm{~cm}$ downstream from the IBM gap with an 85-cm long ferroelectric plasma source [36]. A filtered cathodic arc plasma source was also used for neutralizing the beam at the diagnostic plane [37]. The plasma number density has been measured in the range of $10^{10}-10^{11} \mathrm{~cm}^{-3}$. The beam current was measured using a pinhole Faraday cup with a 1-ns time response [15]. Hole plates are used with these Faraday cups to screen out plasma electrons and ions from confounding the measurement of the beam current. Note that the 1-ns response time (pulse width measurements have an uncertainty of roughly $\pm 0.8 \mathrm{~ns}$ ) of the device is barely sufficient to measure the 1 ns compressed ion beam pulses we predict from the $2 \mathrm{D}$ integrated simulations. Measurement of the beam transverse intensity profile was determined from beam-induced light emission from an intercepting alumina scintillator.

The simulations used the experimental magnetic tune, measured Marx and IBM waveforms, and a kinetic plasma model. They do not include 3D effects or have sufficient

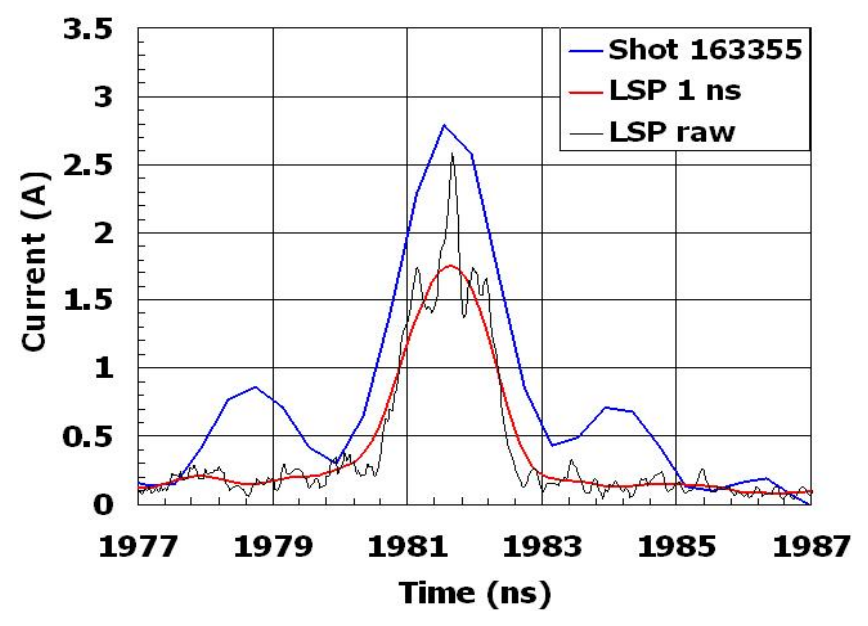

FIG. 7. (Color) The current near compression at $z=414 \mathrm{~cm}$ is plotted for the measured NDCX (shot 163355) and 2D integrated LSP simulation with a 1-ns Gaussian response and the raw LSP data.

resolution to adequately model two-stream instability growth, so these results should be considered optimistic. Consistent with the measurements, we assume an initially uniform 3-eV, $3 \times 10^{10}-\mathrm{cm}^{-3}$ plasma.

The comparison of the measured and simulated currents at the plane of measured simultaneous focus $(z=414 \mathrm{~cm})$ is shown in Fig. 7. The experimental signal from the Faraday cup was time shifted to correspond with the maximum of the LSP result. The raw simulated current along with a 1-ns FWHM Gaussian response folded into the signal is plotted to model the detector response. The FWHM of the folded LSP signal (1.65 ns) is still narrower than that of the experiment ( $2 \mathrm{~ns}$ ) but is well within the 0.8ns measurement error bars. In other measurements, FWHM as small as $1.6 \mathrm{~ns}$ have been measured on NDCX, but the measurement of a still smaller pulse is limited by the 1-ns detector response. Peak compression ratios are both $\approx 100$, however the folded LSP signal has a

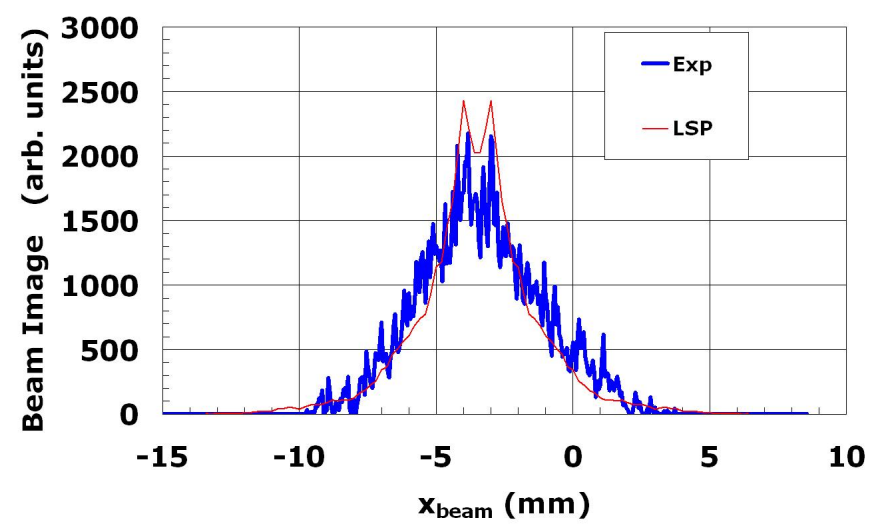

FIG. 8. (Color) The beam current density in the $x$ plane integrated over all $y$ is plotted for the NDCX measured (scanned on April 19, 2007) and LSP simulation values at $z=414 \mathrm{~cm}$. 
reduced CR of 67. The larger measured FWHM and CR are suggestive of a longer effective IBM pulse width than simulated.

The measured and simulated transverse beam current profiles (profiles are plotted versus $x$ integrated for all $y$ ) are compared in Fig. 8. The LSP profile is indicative of a smaller spot size $(3.0 \mathrm{~mm}$ at $z=414 \mathrm{~cm}$, but the beam spot reaches a $1.3-\mathrm{mm}$ minimum at a slightly different plane) than the measured value $(3.8 \mathrm{~mm})$, but exhibits a remarkably similar shape. Here as discussed in Sec. III B, differences can be attributed to experimental beam offsets that limited the spot size since the LSP simulation was 2D. These comparisons are encouraging and demonstrate the utility of the source-to-target simulation capability.

\section{SUMMARY AND CONCLUSIONS}

A numerical investigation using the electromagnetic LSP code into the limiting physics of simultaneous beam focus on the NDCX experiment has shown an overall compression of the ion beam density from the IBM gap to focus of 8000. Given an ion source temperature of $0.1 \mathrm{eV}$ at the anode, longitudinal cooling of the beam as it accelerates in the diode produces $T_{\|}<0.01 \mathrm{eV}$. The space-charge potential, beam focusing angle, and two-stream interaction all contribute additional axial heating of order $0.01 \mathrm{eV}$. Because the beam acceleration time in the diode is roughly that of the effective IBM pulse width, $T_{\|}$is relatively insensitive to Marx voltage errors of order $1 \%$. At a given axial plane, the longer frequency fluctuations (if of random phase) can move the plane of peak axial compression, producing shot-to-shot variation in compression which might impact the interpretation of target heating experiments. As the effective IBM pulse width is increased in upcoming experiments, more care in tuning the Marx voltage might be necessary to reduce this variability. Instability in the solenoid transport section (Harris-like instability) is largely benign for the NDCX conditions with little increase in the longitudinal emittance calculated. Thus, the calculated longitudinal compression ratios of 120 , constrained by the accuracy of the IBM waveform, are reasonable and largely confirmed in experiment.

The integrated simulations calculate a transverse emittance of $0.08 \pi$-mm-mrad which again is consistent with experimental measurement and the cold ion source temperature. The calculated minimum spot size of $1.3 \mathrm{~mm}$ and maximum energy deposition of $0.046 \mathrm{~J} / \mathrm{cm}^{2}$ are insensitive to plasma density greater than $10^{10} \mathrm{~cm}^{-3}$. Compressed beam densities near the target can be greater than the plasma density in kinetic plasma simulations due to the short duration of the compressed beam density and shallow focusing angle.

Comparison of 2D integrated simulations with experimental measurements on NDCX shows good qualitative agreement. The simultaneous focal plane in each case was roughly $z=414 \mathrm{~cm}$. At the measure plane of simulta- neous focus, the measured pulse width is somewhat larger than that simulated ( 2 ns verses $1.3 \mathrm{~ns}$ ), but, within $20 \%$, give the same compression ratio. A detector with faster temporal response than 1 ns may be required for more accurate comparison with simulation. The simulated transverse spot size is within $21 \%$ of the experimental value. Simulations suggest that accelerator alignment is a key issue for the experiment and could easily explain the difference in transverse spot size. Because of the voltage rise in the IBM, beam offsets entering the IBM gap are magnified by roughly 2 at the focal plane. A $5-\mathrm{mm}$ beam offset degrades the energy deposition by 55\%. To reliably heat a target placed at the focal plane, keeping misalignments to submillimeter is prudent and is currently (using magnetic dipoles in the magnetic section) being approached in experiment.

\section{ACKNOWLEDGMENTS}

The authors would like to thank Grant Logan, Ron Davidson, Simon Yu, Alex Friedman, and Craig Olson for years of guidance and support. This work was funded by the Department of Energy through Princeton Plasma Physics Laboratory.

\section{APPENDIX A: SEMIANALYTIC OPTIMIZATION OF ION BEAM COMPRESSION}

With a semianalytic procedure, an optimal tilt voltage waveform can be found for an incoming beam that includes finite tilt gap width and beam space-charge effects. We consider the geometry shown schematically in Fig. 9. A voltage is applied across a gap of effective width $g=d+$ $R$ in a cylindrical drift tube of radius $b$. Beyond position $z=h$, the tube is filled with plasma sufficient to completely neutralize the ion beam space charge. Including the effects of finite gap width, as well as beam space-charge spreading, we attempt to find a temporal voltage profile $V(t)$ that produces a longitudinal focus at $L$ for a cold ion beam. We first describe an analytic form developed by Lee [38] of the on-axis electric field profile of the gap shown in Fig. 10. We then present a model for the longitudinal space-charge forces within the beam. Results from the

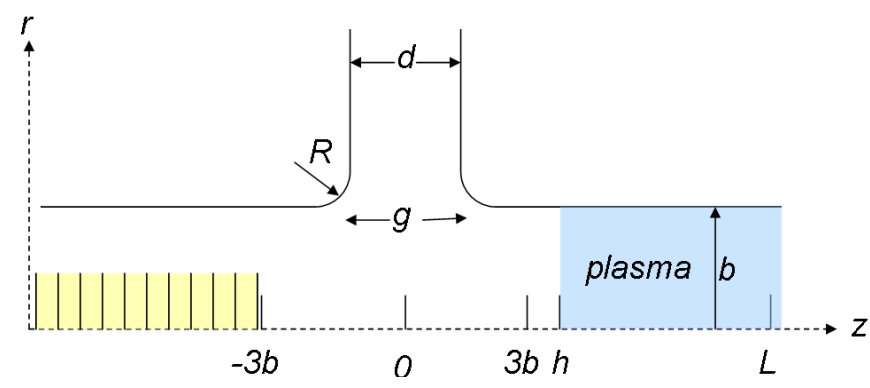

FIG. 9. (Color) Schematic of the incoming beam, drift tube, IBM gap, and plasma geometry used in the semianalytic optimization model. 


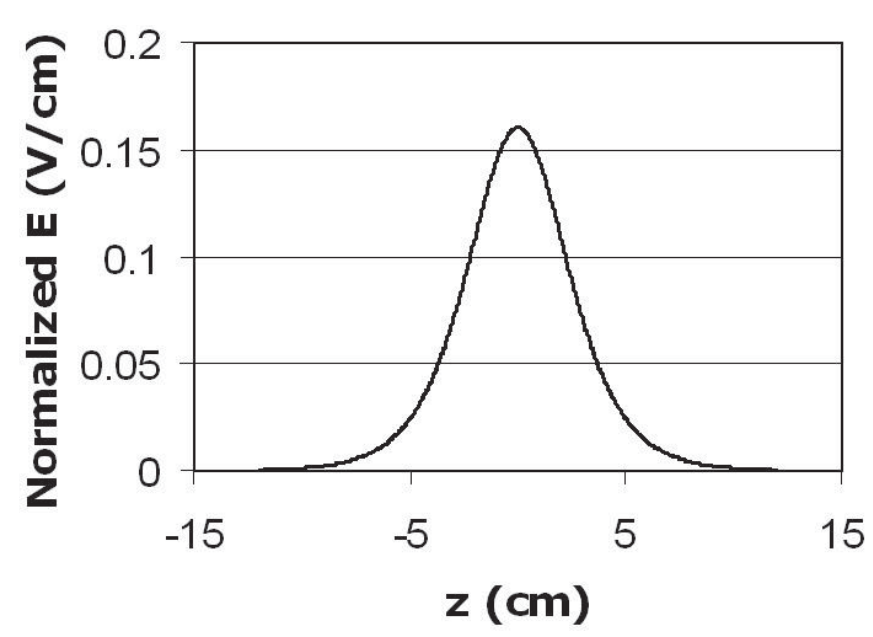

FIG. 10. The longitudinal electric field for the IBM gap model [Eq. (A8)] with $b=g=4 \mathrm{~cm}$.

numerical transport calculations for a 200-ns, 27-mA K $\mathrm{K}^{+}$ NDCX beam are then discussed.

\section{Electrostatic field from an axisymmetric gap}

We now describe the form of the axial electric field as first proposed by Lee [38]. If the electric field $E_{z}$ is known at $r=b$ for all $z$, then it can be determined for $r<b$ by a Fourier integral transform. We write

$$
E_{z}^{F}(r, k)=\int_{-\infty}^{\infty} d z e^{-i k z} E_{z}(r, z)
$$

and note that from Gauss's Law $E_{z}^{F}$ satisfies the $m=0$ modified Bessel equation so that

$$
E_{z}^{F}(r, k)=\frac{I_{0}(k r)}{I_{0}(k b)} E_{z}^{F}(b, k) .
$$

The inverse transform of Eq. (A1) then gives

$$
E_{z}(r, z)=\int_{-\infty}^{\infty} d k \frac{e^{i k z} I_{0}(k r)}{2 \pi I_{0}(k b)} E_{z}^{F}(b, k)
$$

Fortunately small scale variations in $E_{z}$ near the wall at $r=$ $b$ have little impact near the axis, so we can guess a simple approximate form for $E_{z}(b, z)$ and use it to obtain a formula which is accurate near $r=0$. For a gap with small corner radius of curvature $(R \ll d)$, we take

$$
E_{z}(b, z) \simeq \begin{array}{cc}
V / g & |z|<g / 2 \\
0 & |z|>g / 2
\end{array}
$$

Substituting in Eq. (A1) gives

$$
E_{z}^{F}(b, k)=\frac{2 V}{k g} \sin \left(\frac{k g}{2}\right),
$$

and from Eq. (A3) we get

$$
E_{z}(r=0, z) \simeq \frac{V}{\pi b} \int_{0}^{\infty} d x \frac{\cos \left(\frac{x z}{b}\right)}{I_{0}(x)} \frac{\sin \left(\frac{x g}{2 b}\right)}{(x g / 2 b)},
$$

where we have made the substitution $x=k b$ and made use of system symmetry about the gap center. In the infinitesimal gap limit $g \rightarrow 0$, the following approximation of Eq. (A6) is accurate to a maximum relative error of $\pm 2 \times$ $10^{-4}$

$$
E_{0}(z) \simeq \frac{V}{b J_{1}\left(x_{1}\right)\left[2 \cosh \left(x_{1} z / s b\right)\right]^{2}},
$$

where $x_{1}$ is the first zero of $J_{0}(x)$ and $s=1.538781$. A finite gap can be well approximated as a series of short gaps each creating a field of the infinitesimal gap form above but displaced in $z$ and with the appropriate voltage drop. For the model calculations carried out here, $b=g=4 \mathrm{~cm}$, a three term approximation

$$
E_{z}(0, z) \simeq \sum_{n=1}^{3} \frac{1}{3} E_{0}\left[z-\frac{(n-2) g}{3}\right]
$$

gives an excellent fit to Eq. (A6). Truncation of the spatial field profile at \pm 3 tube radii and proper renormalization, i.e.,

$$
\int_{-3 b}^{3 b} E_{z}(0, z) d z=1
$$

introduces negligible error. $E_{z}(0, z)$ is plotted in Fig. 10. We make use of this particular form in the following calculations.

\section{Treatment of beam space-charge forces}

We consider a semi-infinite grounded cylindrical tube defined by the conducting surfaces at $r=b$ and $z=h$ (the plasma is considered at ground). The electrostatic potential for a disk of radius $a$, width $w$, and uniform charge density $n_{0} e$ located on-axis at $z^{\prime}=z_{i}$ is

$$
\phi_{i}(r, z)=\int_{z_{i}-w / 2}^{z_{i}+w / 2} d z^{\prime} n_{0} e \int_{0}^{a} 2 \pi r^{\prime} d r^{\prime} \bar{\phi}\left(r, z, r^{\prime}, z^{\prime}\right)
$$

where the Green's function $\bar{\phi}$ is given by

$$
\bar{\phi}\left(r, z, r^{\prime}, z^{\prime}\right)=\frac{4}{b} \sum_{n=1}^{\infty} \frac{J_{0}\left(x_{0 n} r / b\right) J_{0}\left(x_{0 n} r^{\prime} / b\right) e^{x_{0 n}\left(z_{<}-h\right) / b} \sinh \left[x_{0 n}\left(h-z_{>}\right) / b\right]}{x_{0 n} J_{1}^{2}\left(x_{0 n}\right)} .
$$

Here, $z_{<}\left(z_{>}\right)$is the lesser (greater) of $z$ and $z^{\prime}$ and the $x_{0 n}$ are the zeros of the Bessel function $J_{0}(x)$. For simplicity, we consider only the on-axis potential and perform the integration in Eq. (A10) over $r^{\prime}$ to get 


$$
\phi_{i}(0, z)=\sum_{n=1}^{\infty} \frac{8 \pi n_{0} e a J_{1}\left(x_{0 n} a / b\right)}{\left[x_{0 n} J_{1}\left(x_{0 n}\right)\right]^{2}} \int_{z_{i}-w / 2}^{z_{i}+w / 2} d z^{\prime} e^{x_{0 n}\left(z_{<}-h\right) / b} \sinh \left[x_{0 n}\left(h-z_{>}\right) / b\right]
$$

The integrations in Eq. (A12) are carried out separately for cases $z<z_{i}-w / 2,\left|z-z_{i}\right|<w / 2$ and $z>z_{i}+w / 2$. The total on-axis electric field is obtained by summing over the disks $i$,

$$
E_{z}(z)=-\sum_{i} \frac{\partial \phi_{i}(0, z)}{\partial z}
$$

For the model calculations below ( $b=4 \mathrm{~cm}$ and $a=$ $1 \mathrm{~cm}$ ), 10-12 terms in the sum found in Eq. (A13) provide adequate convergence.

\section{Numerical calculations}

Numerical calculations are carried out for a nominal set of NDCX beam parameters: 300-keV energy, 27-mA current, 200-ns pulse length, focal length $L=130 \mathrm{~cm}$ and 1$\mathrm{cm}$ radius which gives $n_{0}=4.48 \times 10^{8} \mathrm{~cm}^{-3}$. The $200-\mathrm{ns}$ pulse is divided into 20 disks for the numerical transport. The head of the pulse is assumed to arrive at position $z=$ $-3 b$ (where gap field begins) at $t=0$ or the time at which the gap voltage begins (see Fig. 9). The equation of motion for each disk is integrated forward in time using a simple predictor-corrector finite time difference algorithm with a time step of 13 ps.

If we ignore space-charge forces and make use of Eq. (1), the beam is focused at $z=L$ by the temporal IBM voltage profile,

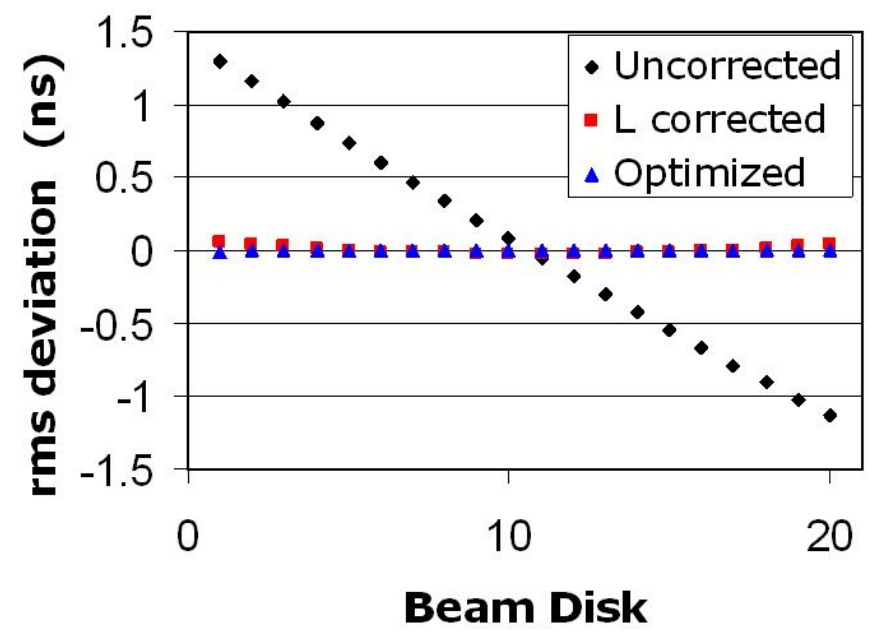

FIG. 11. (Color) Considering only the finite gap effect with $b=$ $g=4 \mathrm{~cm}$, the rms deviation of arrival time from the mean is plotted for the beam disks (disks 1-20). The model results are plotted using $V(t)$ from Eq. (A14) (uncorrected), corrected by adjusting the focal length $L$ ( $L$ corrected), and fully optimized using least squares minimization (optimized).

$$
V(t)=\frac{m_{i}}{2 e}\left\{\left[\frac{v(0)}{1-v(0) t / L}\right]^{2}-v(0)^{2}\right\}
$$

where $v(0)$ is the velocity of the beam head exiting the gap. In these calculations for a $200-n s$ pulse, the initial bias to the gap is $-100 \mathrm{kV}$ giving the beam head a $200-\mathrm{keV}$ energy and $v(0)=0.00332 c$. This voltage profile is used with the spatial variation of $E_{z}$ discussed in Sec. A 1 . The equation of motion for each disk is integrated forward in time until the pulse centroid reaches $z=L$. The resulting distribution of disk positions and velocities is shown in Fig. 11. The rms temporal spread in the arrival time at $L$ is $0.74 \mathrm{~ns}$. The effect of the finite gap width is to shift the focal plane downstream and to introduce a small velocity variation due to the varying disk transit times across the gap. Shown in Fig. 11, using $L=128.33 \mathrm{~cm}$ produced the correct mean arrival time but left a residual 24-ps rms temporal spread. An additional voltage correction of the form $\Delta V(t)=p_{2}\left(t-p_{1}\right)+p_{3}\left(t-p_{1}\right)^{2}$ is added with the parameters $p_{i}$ determined from Powell least squares minimization. The resulting distribution of disk arrival time, also shown in Fig. 11, has a reduced rms spread of $\sim 5 \mathrm{ps}$ (of order half the numerical time step). The voltage correction $\Delta V(t)$ [in addition to that of Eq. (A14)] required to produce the optimized temporal distribution has a maximum value of less than $3 \mathrm{kV}$ at the end of the 200-ns pulse (see Fig. 12).

In calculating the effect of the beam space-charge repulsion, $100 \mathrm{~ns}$ of sacrificial beam is added to the head and tail of the 200-ns pulse to more accurately model the NDCX experiment which actually has a pulse length $>5 \mu \mathrm{s}$. These added beam disks essentially screen the

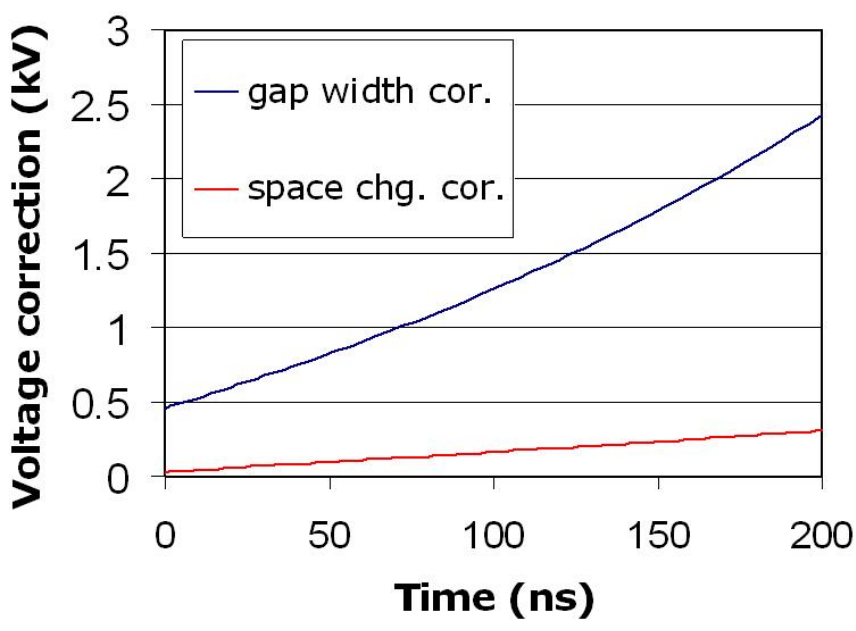

FIG. 12. (Color) Optimized IBM voltage correction $\Delta V(t)$ for finite gap width and space charge for the NDCX beam with $b=$ $g=4 \mathrm{~cm}$. 
beam pulse from significant longitudinal distortions. Only after the beam density has been modified by the applied tilt voltage between the gap and the plasma does the beam space charge have a significant effect on the longitudinal dynamics.

The transport calculations are repeated with the spacecharge model discussed in Sec. A 2. Using the voltage temporal profile optimized for the finite gap effect without space charge leaves a residual rms temporal spread of $0.15 \mathrm{~ns}$. The effect is greatest in the beam head due the sacrificial beam disks in the front slightly outrunning the optimized 200-ns pulse. Optimization of the parameters $L$ and $p_{i}$ again using the least squares minimization reduces the rms spread to $23 \mathrm{ps}$. The added voltage corrections to $\Delta V(t)$ for the space charge are also shown in Fig. 12. Although these corrections are small for the long pulse NDCX experiment, the impact of space charge can quickly become significant as the total beam pulse width becomes comparable to the compressed pulse.

For the 27-mA, 200-ns beam pulse with the sacrificial current in front and behind, the effects of finite gap width and space charge are relatively small and to the precision of the calculation are correctable. The total maximum voltage correction required for optimization including both effects was $3 \mathrm{kV}$. An rms spread of $23 \mathrm{ps}$ is obtained which is of the order of the numerical time step. This value is $1.5 \%$ of the total tilt voltage of $\pm 100 \mathrm{kV}$ used in these NDCX calculations. Thus, with corrections, these effects are quite a bit smaller than that of beam thermal spreading.

[1] B. G. Logan et al., Nucl. Fusion 45, 131 (2005).

[2] S. S. Yu et al., Nucl. Fusion 47, 721 (2007).

[3] R. O. Bangerter, Nucl. Instrum. Methods Phys. Res., Sect. A 415, 3 (1998).

[4] D. A. Callahan, Fusion Eng. Des. 32-33, 441 (1996).

[5] B. G. Logan and D. A. Callahan, Nucl. Instrum. Methods Phys. Res., Sect. A 415, 468 (1998).

[6] D. R. Welch, D. V. Rose, B. V. Oliver, and R. E. Clark, Nucl. Instrum. Methods Phys. Res., Sect. A 464, 134 (2001).

[7] S.S. Yu et al., in Proceedings of the 2003 Particle Accelerator Conference, Portland, OR, edited by J. Chew (IEEE, Piscataway, NJ, 2003), p. 98.

[8] E. Henestroza et al., Phys. Rev. ST Accel. Beams 7, 083501 (2004).

[9] P. K. Roy et al., Phys. Plasmas 11, 2890 (2004).

[10] B. G. Logan et al., Nucl. Fusion 45, 131 (2005).

[11] C. Thoma, D. R. Welch, S. S. Yu, E. Henestroza, P. K. Roy, S. Eylon, and E.P. Gilson, Phys. Plasmas 12, 043102 (2005).

[12] P. K. Roy et al., Nucl. Instrum. Methods Phys. Res., Sect. A 544, 225 (2005).
[13] D. R. Welch, D. V. Rose, T. C. Genoni, S. S. Yu, and J. J. Barnard, Nucl. Instrum. Methods Phys. Res., Sect. A 544, 236 (2005).

[14] P. K. Roy et al., Phys. Rev. Lett. 95, 234801 (2005).

[15] A. B. Sefkow, R. C. Davidson, P. C. Efthimion, and E. P. Gilson, Phys. Rev. ST Accel. Beams 9, 052801 (2006).

[16] LSP is a software product of ATK Mission Research.

[17] D. R. Welch, D. V. Rose, R.E. Clark, T. C. Genoni, and T. P. Hughes, Comput. Phys. Commun. 164, 183 (2004).

[18] D. Baca, J. W. Kwan, J. K. Wu, and E. Chacon-Golcher, in Proceedings of the 2003 Particle Accelerator Conference, Portland, OR, Ref. [7], pp. 3294-3296.

[19] See M. Reiser, Theory and Design of Charged Particle Beams (Wiley, New York, 1994).

[20] R. J. Briggs, in Advances in Plasma Physics, edited by A. Simon and W. B. Thompson (Wiley Interscience, New York, 1971), Vol. 4, p. 43.

[21] T. C. Genoni, D. V. Rose, D. R. Welch, and E. P. Lee, Phys. Plasmas 11, L73 (2004).

[22] D. V. Rose, T. C. Genoni, D. R. Welch, and E. P. Lee, Nucl. Instrum. Methods Phys. Res., Sect. A 544, 389 (2005).

[23] E. A. Startsev and R.C. Davidson, Phys. Plasmas 13, 062108 (2006).

[24] D. V. Rose, T. C. Genoni, D. R.Welch, E. A. Startsev, and R. C. Davidson, Phys. Rev. ST Accel. Beams 10, 034203 (2007).

[25] E. A. Startsev, R. C. Davidson, and H. Qin, Phys. Plasmas 9, 3138 (2002).

[26] E. A. Startsev, R. C. Davidson, and H. Qin, Laser Part. Beams 20, 585 (2002).

[27] E. A. Startsev, R. C. Davidson, and H. Qin, Phys. Rev. ST Accel. Beams 6, 084401 (2003).

[28] E. A. Startsev, R. C. Davidson, and H. Qin, Nucl. Instrum. Methods Phys. Res., Sect. A 544, 125 (2005).

[29] E. A. Startsev, R. C. Davidson, and H. Qin, in Proceedings of the 2005 Particle Accelerator Conference (IEEE, Piscataway, NJ, 2005), p. 558.

[30] E. A. Startsev, R. C. Davidson, and H. Qin, Phys. Rev. ST Accel. Beams 8, 124201 (2005).

[31] E. A. Startsev, R. C. Davidson, and H. Qin, Phys. Plasmas 14, 056705 (2007).

[32] E. G. Harris, Phys. Rev. Lett. 2, 34 (1959).

[33] D. R. Welch et al., Nucl. Instrum. Methods Phys. Res., Sect. A 577, 231 (2007).

[34] D. R. Welch, D. V. Rose, W. M. Sharp, C. L. Olson, and S.S. Yu, Laser Part. Beams 20, 621 (2002).

[35] J.E. Coleman, D. Ogata, P. A. Seidl, P. K. Roy, E.P. Gilson, A. B. Sefkow, and D. R. Welch, in Proceedings of the 2007 Particle Accelerator Conference Albuquerque, NM (IEEE, Piscataway, NJ, 2007).

[36] P. C. Efthimion et al., Nucl. Instrum. Methods Phys. Res., Sect. A 544, 378 (2005).

[37] A. Anders and G. Y. Yushkov, J. Appl. Phys. 91, 4824 (2002).

[38] E. P. Lee (private communication). 